\title{
Have we gone too far? Endovascular stent-graft repair of aortobronchial fistulas
}

Grayson H. Wheatley III, MD, a Anthony Nunez, MD, ${ }^{\text {b }}$ Ourania Preventza, MD, ${ }^{a}$ Venkatesh G. Ramaiah, MD, ${ }^{a}$ Julio A. Rodriguez-Lopez, MD, ${ }^{a}$ James Williams, MD, ${ }^{a}$ Dawn Olsen, $\mathrm{PA}-\mathrm{C}_{\text {, }}{ }^{\mathrm{a}}$ and Edward B. Diethrich, $\mathrm{MD}^{\mathrm{a}}$

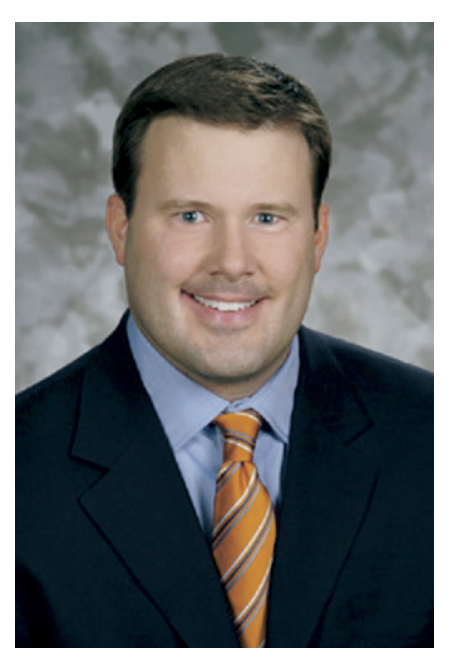

Dr Wheatley
Objective: Although endovascular repair of the descending thoracic aorta has emerged as a viable treatment option, little is known about its potential to treat patients diagnosed with aortobronchial fistulas. We reviewed our comprehensive thoracic endografting experience with regard to the endovascular management and subsequent outcome of patients with aortobronchial fistulas to assess whether endoluminal graft repair is a realistic option.

Methods: Between February 2000 and November 2005, 255 patients were successfully treated with an endoluminal graft to the descending thoracic aorta. Indications for intervention included: atherosclerotic aneurysms (109/255, 42.7\%), acute and chronic dissections $(75 / 255,29.4 \%)$, miscellaneous $(34 / 255,13.3 \%)$, penetrating aortic ulcers $(30 / 255,11.8 \%)$, and aortobronchial fistulas $(7 / 255,2.7 \%)$.

Results: Average patient age was $73.4 \pm 10.1$ years, with 4 male patients (4/7, $57.1 \%)$ and 3 female patients $(3 / 7,42.9 \%)$. All patients presented with hemoptysis, with 1 patient $(1 / 7,14.3 \%)$ requiring preoperative blood transfusion. Three patients $(3 / 7,42.9 \%)$ were diagnosed with atherosclerotic aneurysms, 3 patients $(3 / 7,42.9 \%)$ had pseudoaneurysms associated with prior open surgical repair, and 1 patient (1/7, $14.3 \%$ ) had a prior endoluminal graft placed for a traumatic aortic transection. No standard postoperative antibiotic regimen was followed. There were no endoleaks, no incidences of paraplegia, and no endoluminal graft infections. Survival was $100 \%$ (7/7) at both 30 days and 1 year, and all patients are currently alive. Follow-up computed tomography was available for all 7 patients, with an average follow-up of $42.6 \pm 28.5$ months.

Conclusions: Endovascular management of aortobronchial fistulas appears to be safe and well tolerated, even in surgically high-risk patients, with minimal risk of prosthesis infection. Long-term surveillance and continued investigation are warranted.

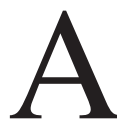
ortobronchial fistulas (ABFs) can be associated with a number of pathologic conditions of the descending thoracic aorta (DTA), including atherosclerotic aneurysms, para-anastomotic pseudoaneurysms secondary to previous open surgical repair, and mycotic aneurysms. ${ }^{1-3}$ In addition, ABFs have been associated with invasive aspergillus infection of the lung, unilateral lung transplantation, and pulmonary tuberculosis. ${ }^{4-6}$ Usually presenting as massive and/or intermittent hemoptysis, a high index of suspicion and early diagnosis are critical. Although rare, this life-threatening disorder requires urgent intervention and treatment. The operative mortality for traditional open surgical repair of ABF using the clamp-and-sew technique reaches $20 \%$ in the modern era. ${ }^{7}$

As endovascular repair of the DTA is emerging as a viable treatment option for uncomplicated atherosclerotic aneurysms, little is known about its potential to treat patients diagnosed with ABF. Currently, only 1 thoracic endoprosthesis has gained 

Abbreviations and Acronyms
$\mathrm{ABF}=$ aortobronchial fistula
$\mathrm{CFA}=$ common femoral artery
$\mathrm{CT}=$ computed tomography
DTA $=$ descending thoracic aorta
ELG $=$ endoluminal graft
IDE = investigator device exemption
TEE $=$ transesophageal echocardiogram

approval by the United States Food and Drug Administration for endovascular treatment of the DTA. Use of this device is limited to atherosclerotic aneurysms; however, several case reports have been published describing endovascular stent-graft repair of $\mathrm{ABF}$ using several different types of thoracic endoprostheses. ${ }^{8-10}$ The results have been, in general, favorable. We reviewed our comprehensive thoracic endografting experience with regards to the endovascular management and subsequent outcome of patients with $\mathrm{ABF}$ to assess whether endovascular stent-graft repair is a realistic option.

\section{Materials and Methods}

Between February 2000 and November 2005, 255 consecutive patients with diverse pathologies of the DTA were prospectively enrolled in an Arizona Heart Hospital Institutional Review Boardapproved single-site investigational device exemption clinical study. All patients were treated with TAG endoprostheses (W. L. Gore \& Associates, Flagstaff, Ariz) under the investigational protocol and were fully informed and consented prior to implantation of the endoluminal graft (ELG). Enrollment in the study was limited to patients who were deemed to be at "high surgical risk," meaning that they had comorbidities and/or thoracic aortic pathologies that placed them at prohibitive risk for open repair, and, without intervention, an adverse event could be anticipated within days or weeks. During this study period, no patients were treated nonoperatively or with open surgery.

Seven patients with ABF were treated (Table 1). Mean age was $73.4 \pm 10.1$ years, with 4 male patients $(4 / 7,57.1 \%)$ and 3 female patients $(4 / 7,42.9 \%)$. All patients with ABF presented with hemoptysis $(n=7)$. Patients with ABF were diagnosed preoperatively based upon clinical findings of hemoptysis and diagnostic tests, which included chest radiography $(n=7)$, contrast-enhanced computed tomography (CT) angiography $(\mathrm{n}=7)$, bronchoscopy ( $=5)$, angiography $(\mathrm{n}=3)$, and transesophageal echocardiography $(n=3)$. All patients were hemodynamically stable at the time of their intervention.

Following discharge, patients were seen within 2 weeks to assess the incision sites and to evaluate the patient's overall health status. CT scans, plain radiographs, and physical examinations were obtained at 1,6 , and 12 months and yearly thereafter. All significant medical events that resulted in either an unplanned increase in the level of care, permanent sequela, or death were recorded.

\section{Results}

The TAG endoprosthesis was successfully delivered and deployed in all 7 patients $(7 / 7,100 \%)$, with no open thoracic conversions. Adequate ELG placement and exclusion of the ABF was confirmed by completion angiography. Ten ELGs were deployed in 7 patients. Table 2 lists the sizes of the ELGs deployed along with the method of vascular access for sheath delivery. In 2 patients, the external iliac artery was too small to accommodate the delivery sheath; a retroperitoneal incision was performed, and a 10-mm Dacron conduit was anastomosed to the proximal common iliac artery. The conduit was then used to deliver both the sheath and the device. In 2 patients, the conduit was ligated, and in 1 patient the conduit was tunneled to the ipsilateral common

TABLE 1. Characteristics of patients with aortobronchial fistulas treated with an endoluminal graft

\begin{tabular}{|c|c|c|c|}
\hline Age $(y) / \operatorname{sex}$ & Aortic pathology & Symptoms & Comorbidities \\
\hline $52 / \mathrm{M}$ & $\begin{array}{l}\text { Pseudoaneurysm; prior DTA aneurysm } \\
\text { repair } \times 2\end{array}$ & Hemoptysis & Active tobacco use \\
\hline $75 / \mathrm{M}$ & $\begin{array}{l}\text { Pseudoaneurysm, prior DTA aneurysm } \\
\text { repair, new DTA aneurysm, } \\
\text { abdominal aortic dissection }\end{array}$ & Hemoptysis & $\begin{array}{l}\text { Type II DM, HTN, aortic valve replacement, CHF, } \\
\text { atrial fibrillation }\end{array}$ \\
\hline $75 / F$ & $\begin{array}{l}\text { Pseudoaneurysm, prior DTA aneurysm } \\
\text { repair }\end{array}$ & Hemoptysis & $\begin{array}{l}\text { HTN, COPD, MI, CVA, CHF, severe Al, ulcerative } \\
\text { colitis, home oxygen }\end{array}$ \\
\hline $77 / F$ & DTA aneurysm & Hemoptysis & Renal insufficiency, COPD, active tobacco use \\
\hline $75 / \mathrm{M}$ & DTA aneurysm, penetrating aortic ulcer & Hemoptysis & $\begin{array}{l}\text { HTN, prior abdominal aortic aneurysm repair with } \\
\text { ELG, peripheral vascular disease }\end{array}$ \\
\hline $71 / \mathrm{F}$ & DTA aneurysm & Hemoptysis & $\begin{array}{l}\text { HTN, COPD, prior ascending aortic aneurysm } \\
\text { repair, active tobacco use }\end{array}$ \\
\hline $69 / \mathrm{M}$ & $\begin{array}{l}\text { Prior thoracic ELG for aortic transection } \\
\text { (6 y prior) }\end{array}$ & Hemoptysis & $\begin{array}{l}\text { MVA, HTN, aortic transection, multiple abdominal } \\
\text { surgeries, hepatitis B }\end{array}$ \\
\hline
\end{tabular}

$D M$, Diabetes mellitus; $H T N$, hypertension; $C H F$, congestive heart failure; COPD, chronic obstructive pulmonary disease; $M I$, myocardial infarction; $A l$, aortic insufficiency; CVA, cerebral vascular accident; MVA, motor vehicle accident; DTA, descending thoracic aorta; ELG, endoluminal graft. 
TABLE 2. Number and sizes of thoracic endoprostheses used with vascular access

\begin{tabular}{cccc}
\hline Patient \# & Number of endoprostheses & Endoprostheses sizes & Vascular access \\
\hline 1 & 1 & $28 \mathrm{~mm} \times 7 \mathrm{~cm}$ & Common femoral artery \\
2 & 2 & $34 \mathrm{~mm} \times 20 \mathrm{~cm}$ & Common femoral artery \\
3 & 1 & $40 \mathrm{~mm} \times 10 \mathrm{~cm}$ & Retroperitoneal conduit \\
4 & 1 & $34 \mathrm{~mm} \times 20 \mathrm{~cm}$ & Retroperitoneal conduit \\
5 & 1 & $40 \mathrm{~mm} \times 15 \mathrm{~cm}$ & Common femoral artery \\
6 & 2 & $37 \mathrm{~mm} \times 20 \mathrm{~cm}$ & Common femoral artery \\
7 & 2 & $37 \mathrm{~mm} \times 10 \mathrm{~cm}$ & Common femoral artery \\
& & $34 \mathrm{~mm} \times 20 \mathrm{~cm}$ & $34 \mathrm{~mm} \times 15 \mathrm{~cm}$ \\
\hline
\end{tabular}

femoral artery (CFA), and an iliac-CFA bypass was performed. In the other 4 patients, vascular access and sheath delivery were safely performed through the CFA. There were no iliac artery ruptures secondary to sheath delivery or removal and no complications associated with vascular access.

To better define the most proximal extent of the landing point for the ELG, the thoracic aorta has been divided into several zones. Zone 1 is the ascending aorta up to and including the origin of the innominate artery; zone 2 includes the origin of the left common carotid artery; zone 3 includes the origin of the left subclavian artery and proximal DTA; and zone 4 is the mid to distal DTA. The ELG was landed in zone 2 in 1 patient with intentional coverage of the left subclavian artery, zone 3 in 5 patients, and zone 4 in 1 patient. In the patient who required coverage of the left subclavian artery to achieve adequate proximal fixation, the subclavian artery was not coil embolized, and no carotid-subclavian bypass was performed. This patient had no signs of arm or hand ischemia and was completely asymptomatic. Neurologically, there were no cerebral vascular accidents or transient ischemic attacks associated with the procedures. There were no incidences of paraparesis or paraplegia. We elected not to utilize spinal drains in any of the patients and maintained increased spinal perfusion by maintaining a systolic blood pressure greater than $140 \mathrm{~mm}$ $\mathrm{Hg}$ for 48 hours postoperatively. We did not monitor spinal cord somatosensory evoked potentials.

Postoperatively, there were no significant episodes of arrhythmias or ST segment changes noted on telemetry monitoring. There were no episodes of chest pain or myocardial infarctions. One patient developed midback and rib pain on postoperative day 1 , which resolved spontaneously by postoperative day 4 . Two patients required blood transfusions with each patient receiving $1 \mathrm{U}$ of packed red blood cells. The average amount of nonionic contrast used for the procedures was $336.4 \pm 206.7 \mathrm{~mL}$ (range $120-640 \mathrm{~mL}$ ). There were no episodes of acute renal insufficiency secondary to contrast nephropathy, and all patients were aggres- sively hydrated intravenously with normal saline solution. One patient had a baseline creatinine level of 2.0 $\mathrm{mg} / \mathrm{dL}$ and was discharged with a creatinine level of 1.9 $\mathrm{mg} / \mathrm{dL}$. This patient received a fenoldopam intravenous drip pre- and postoperatively to assist in minimizing contrast nephropathy.

All patients received a $\mathrm{CT}$ angiogram of the chest, abdomen, and pelvis prior to discharge. There were no endoleaks or ELG migrations detected. All ABFs appeared to have sealed by the time of the CT angiogram, and there was stability of the aortic diameter over time (Figure 1). The hemoptysis resolved in all patients prior to discharge. All patients received intravenous doses of cefazolin perioperatively. No standard antibiotic regimen was implemented. Five patients were discharged home on oral antibiotics, and 2 patients were not discharged on any antibiotics (Table 3 ). There were no clinically documented pneumonias and no signs of infection in any of the patients. The average length of stay in the hospital was $4 \pm 1.1$ days (range 3-6 days).

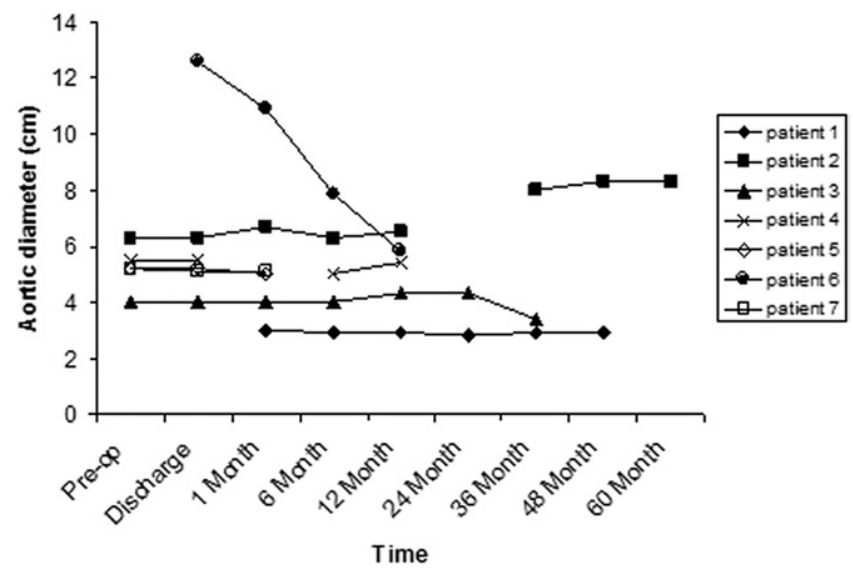

Figure 1. Aortic diameter $(\mathrm{cm})$ in patients with ABFs treated with an ELG preoperatively and at regularly scheduled postoperative follow-up intervals. ABF, Aortobronchial fistula; ELG, endoluminal graft. 
TABLE 3. Antibiotic administration in patients with aortobronchial fistulas treated with an endoluminal graft

\begin{tabular}{|c|c|c|c|}
\hline Patient & Perioperative & Postoperative & Outpatient \\
\hline$\# 1$ & Cefazolin $1 \mathrm{~g}$ IV q8h $\times 3$ doses & $\begin{array}{l}\text { Ceftazidime } 1 \mathrm{~g} \text { IV q12h } \times 4 \text { doses } \\
\text { Ciprofloxicin } 500 \mathrm{~g} \mathrm{PO} \times 1 \text { dose }\end{array}$ & Ciprofloxicin 500 mg PO BID (5 d) \\
\hline$\# 2$ & Cefazolin $1 \mathrm{~g} \mathrm{IV} \mathrm{q8h} \times 3$ doses & None & None \\
\hline$\# 3$ & Cefazolin $1 \mathrm{~g}$ IV q8h $\times 3$ doses & None & None \\
\hline$\# 4$ & Cefazolin $1 \mathrm{~g}$ IV q8h $\times 3$ doses & Vancomycin $1 \mathrm{~g} \mathrm{IV} \times 1$ dose & Cephalexin 500 mg PO QID (7 d) \\
\hline$\# 5$ & Cefazolin $1 \mathrm{~g}$ IV q8h $\times 3$ doses & Levofloxacin $500 \mathrm{mg} \mathrm{IV} \times 2$ dose & Levofloxacin $500 \mathrm{mg}$ PO QD (10 d) \\
\hline \#6 & Cefazolin $1 \mathrm{~g}$ IV q8h $\times 3$ doses & Levofloxacin $500 \mathrm{mg}$ IV $\times 2$ dose & Levofloxacin 500 mg PO QD (7 d) \\
\hline$\# 7$ & Cefazolin $1 \mathrm{~g}$ IV $\mathrm{q} 8 \mathrm{~h} \times 3$ doses & $\begin{array}{l}\text { Ampicillin/sulbactam } 1.5 \mathrm{~g} \mathrm{IV} \mathrm{q8h} \\
\text { (9 doses) }\end{array}$ & Levofloxacin $500 \mathrm{mg}$ PO QD (14 d) \\
\hline
\end{tabular}

$I V$, Intravenous; $P O$, per oral; $B I D$, twice daily; $Q D$, once daily; $Q I D$, four times daily.

There were no deaths and no reinterventions at 30 days postoperatively. Average follow-up was $42.6 \pm 28.5$ months (range 10-75 months). All patients are currently alive $(7 / 7,100 \%)$, and no patient has complained of hemoptysis. There have been no episodes, observed either clinically or by CT scan, of ELG infection. There have been no device fractures or migrations and no endoleaks. There have been no reinterventions.

Only 1 patient required an additional vascular intervention. This patient underwent successful placement of an infrarenal abdominal aortic ELG for an expanding 5-cm abdominal aortic aneurysm 4 years after treatment of the ABF. Three patients have been diagnosed with new medical problems. One patient has developed bladder cancer 5 years after treatment of his $\mathrm{ABF}$, and the bladder cancer is currently controlled with chemotherapy bladder irrigations. Another patient required insertion of a dual-chamber pacemaker for new sick sinus syndrome 2 years following ELG insertion and has also experienced a recent cerebral vascular accident. A third patient has developed a new 2-cm pulmonary nodule, which was detected during routine chest CT imaging for ELG surveillance, and has also developed mild renal insufficiency 3 years following ELG insertion. Three patients have had no new medical problems and are otherwise healthy.

\section{Discussion}

$\mathrm{ABFs}$ represent a challenging therapeutic and diagnostic dilemma. Presenting as either intermittent or massive hemoptysis, mortality is $100 \%$ if intervention is not pursued aggressively. ${ }^{11}$ The fistulous connection results from compression necrosis of the posterior aspect of the left tracheobronchial tree from an associated aneurysm or pseudoaneurysm of the DTA. The size of the fistulous connection determines the magnitude of the hemoptysis. If left untreated, the fistula can continue to enlarge until massive hemoptysis and possibly exsanguination occur. In addition to hemoptysis, additional signs and symptoms of $\mathrm{ABF}$ are dyspnea and cough, chest or back pain, pulmonary rales, or hypoxia. ${ }^{12}$ This disorder can often be confused with pulmonary embolism, pneumonia, or lung abscess, and therefore a high index of suspicion is important.

Several imaging modalities are useful in diagnosing ABF and in differentiating it from other sources of hemoptysis. Chest radiograph, chest CT with intravenous contrast, magnetic resonance imaging, and contrast aortography have all been shown to be successful in demonstrating ABF (Figures $2,3){ }^{2,11}$ In addition, bronchoscopy, intravascular ultrasound, and transesophageal echocardiogram (TEE) are useful modalities for making the diagnosis. Bronchoscopy is particularly useful in ruling out other pulmonary sources of the hemoptysis but should be pursued with caution because of the risk of dislodging any clot and potentially reactivating bleeding. In all of our patients, bronchoscopy and chest CT, radiograph, and aortogram were used to confirm the diagnosis. In addition to assisting with making the diagnosis, both intravascular ultrasound and TEE can assist with intraoperative visualization and localization of the $\mathrm{ABF}$ and help with identifying the appropriate location for stent-graft deployment. We believe that these two imaging modalities

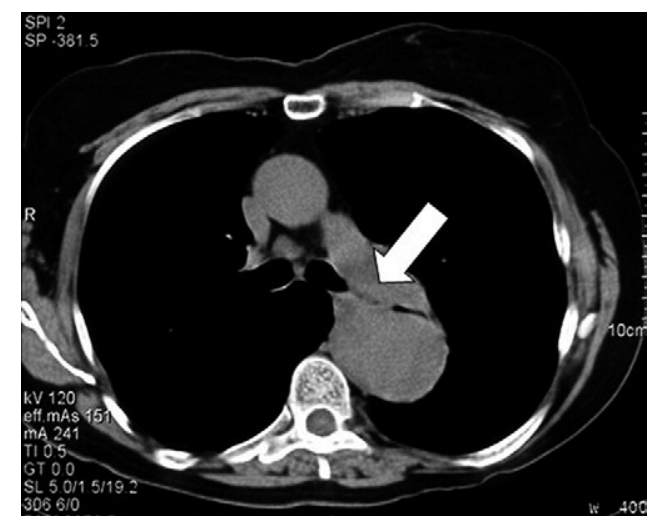

Figure 2. Chest CT demonstrating an ABF (white arrow) from the DTA to the left main stem bronchus. CT, Computed tomographic scan; $A B F$, aortobronchial fistula. 


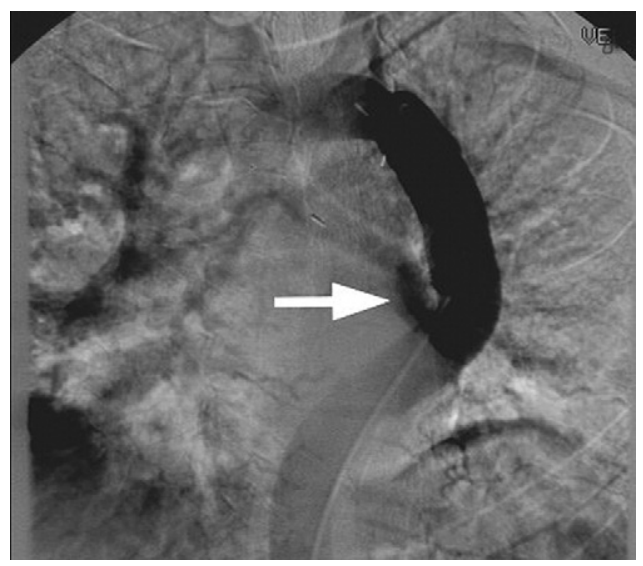

Figure 3. Diagnostic angiogram demonstrating an ABF (white arrow) from the DTA to the left main stem bronchus at the site of a pseudoaneurysm. $A B F$, Aortobronchial fistula; $D T A$, descending thoracic aorta.

are critical for identifying suitable proximal and distal landing zones for the ELG and ensuring that the $\mathrm{ABF}$ is excluded.

Most patients with $\mathrm{ABF}$ have complex aortic pathologic conditions, such as para-anastomotic pseudoaneurysms or sizeable atherosclerotic aneurysms of the DTA, which make open surgical repair a less than straightforward procedure. In addition to the technical challenges of performing a redo left thoracotomy or dealing with a complex DTA aneurysm, the potential exists for prosthetic graft infection. A recent 19-year review of cases of $\mathrm{ABF}$ treated with open surgical repair revealed an operative mortality of $20 \%$ with no associated embolic strokes or paraplegias. ${ }^{7}$ In addition, follow-up with a median of 2.5 years showed no deaths. ${ }^{7}$ Despite improved results with open surgical repair, an opportunity exists to potentially incorporate less invasive technologies, such as endovascular stent-graft repair, into the treatment paradigm for ABF (Figure 4).

A review of the literature shows a total of 36 cases of ABF treated with an ELG (Table $4^{1,2,4,9,13-28}$ ). A metaanalysis demonstrates a cumulative 30-day mortality of $8.3 \%$, with most of the cases reporting successful 1-year survival. In our cohort of 7 patients, we were able to successfully treat the ABF with an ELG in all patients. Although all patients presented with hemoptysis, only 2 patients required a blood transfusion for a hemoglobin value $<10 \mathrm{~g} / \mathrm{dL}$. The relatively low transfusion rate in our patients is attributed to early recognition and intervention, which allowed us to repair the ABF prior to catastrophic hemorrhage and prevent additional blood loss. Survival was $100 \%$ at both 30 days and 1 year. All patients are currently alive up to 75 months postprocedure and have required no reinterventions. Only 1 patient has required a subsequent vas-

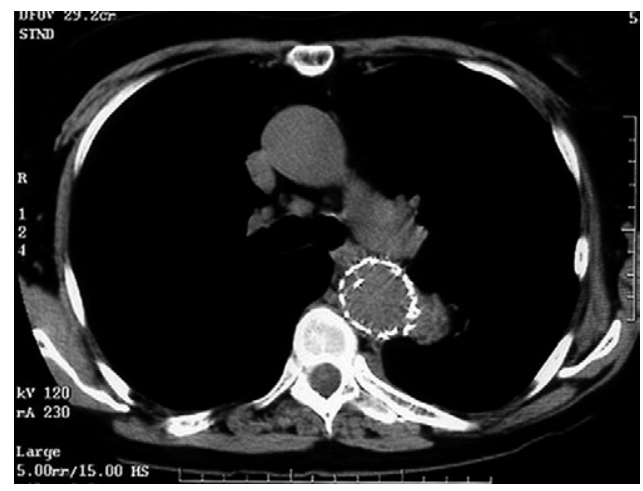

Figure 4. Chest CT demonstrating successful exclusion of an ABF. CT, Computed tomographic scan; $A B F$, aortobronchial fistula.

cular intervention. This patient required ELG repair for an expanding abdominal aortic aneurysm.

We did not utilize spinal cord protection with a spinal drain in any of the patients in this study. In addition, we do not utilize somatosensory evoked potential monitoring for our thoracic endografting procedures. There were no perioperative or postoperative neurologic complications, such as paraplegia or paraparesis. We use a spinal drain selec-

TABLE 4. Meta-analysis of all previously published cases of endoluminal graft repair of aortobronchial fistulas

\begin{tabular}{lcr}
\hline \multicolumn{1}{c}{ Author (year) } & Number of patients & 30-day survival \\
\hline Campagna (1996) & 1 & $1 / 1(100 \%)$ \\
Chuter (1996) & 1 & $1 / 1(100 \%)$ \\
Karmy-Jones (1999) & 1 & $1 / 1(100 \%)$ \\
Miyata (1999) & 2 & $2 / 2(100 \%)$ \\
Dorweller (2001) & 3 & $3 / 3(100 \%)$ \\
Smayra (2001) & 1 & $1 / 1(100 \%)$ \\
Ciati (2001) & 1 & $1 / 1(100 \%)$ \\
Yoo (2001) & 1 & $1 / 1(100 \%)$ \\
Kramer (2001) & 1 & $1 / 1(100 \%)$ \\
Leobon (2002) & 3 & $2 / 3(66 \%)$ \\
Thompson (2002) & $4)^{*}$ & $4 / 4(100 \%)$ \\
Pitton (2002) & 4 & $4 / 4(100 \%)$ \\
Kochi (2002) & 1 & $1 / 1(100 \%)$ \\
Orend (2003) & 1 & $1 / 1(100 \%)$ \\
Bockler (2004) & 8 & $6 / 8(75 \%)$ \\
Numan (2004) & 1 & $1 / 1(100 \%)$ \\
Thalhammer (2004) & 1 & $1 / 1(100 \%)$ \\
Khare (2005) & 1 & $1 / 1(100 \%)$ \\
Islam (2005) & 1 & $1 / 1(100 \%)$ \\
Kaw (2005) & 1 & $1 / 1(100 \%)$ \\
Munneke (2006) & 1 & $1 / 1(100 \%)$ \\
Wheatley (2006) & $4 \dagger$ & $4 / 4(100 \%)$ \\
Total & $36 \ddagger$ & $33 / 36(91.7 \%)$ \\
\hline Two & &
\end{tabular}

*Two patients included in this study. †Four patients included in this study. \$Exclusive of patients already included in this study. 
tively for neurologically high-risk patients, and we place the drain preoperatively in patients with a prior abdominal aortic aneurysm repair (open or endovascular) and/or manipulation of the DTA below the level of T9. In addition, if any patient develops postoperative paraplegia or paraparesis, we will immediately place a spinal drain. Following ELG repair, we help prevent spinal cord ischemia by actively increasing the systolic blood pressure to between 140 and $160 \mathrm{~mm} \mathrm{Hg}$ for 48 hours postoperatively. The subclavian artery is also an important contributor to spinal cord perfusion and collaterals; however, it can frequently be covered without neurologic or vascular compromise. ${ }^{28} \mathrm{We}$ intentionally covered the left subclavian artery in 1 patient in this study to achieve adequate $2-\mathrm{cm}$ proximal fixation of the ELG. We did not perform a preoperative carotidsubclavian bypass in this patient, and there were no neurologic sequelae. In addition, there was no retrograde type II endoleak from the left subclavian artery, and the patient had no signs of vascular insufficiency of the left arm. We have found that it is safe to manage these patients expectantly, and if left arm claudication develops, then we perform an elective carotid-subclavian bypass.

Vascular access is an important consideration for thoracic endografting procedures. Introduction and removal of large-bore delivery sheaths in small, calcified or tortuous access vessels can be associated with rupture of the iliac artery and subsequent patient mortality. It is critical to assess the quality and size of both external and external iliac arteries in patients who are candidates for endovascular repair to see if the arteries will accommodate the large-bore delivery sheath before the patient can be offered endovascular repair. If the access vessels will not accept the largebore delivery sheath, then a $10-\mathrm{mm}$ retroperitoneal conduit must be sewn to the distal aorta or proximal common iliac artery to make the vascular access portion of the procedure safe. In this study, 3 patients $(3 / 7,42.9 \%)$ required a retroperitoneal conduit to safely deliver the sheath. This rate is significantly higher than most series for thoracic endografting. Commonly, $15 \%$ of patients who are treated with a thoracic ELG require a retroperitoneal conduit. ${ }^{29}$ It is unclear why our series has a higher rate of retroperitoneal conduits, but 6 of the 7 patients had aggressive atherosclerotic-related disease as their primary aortic pathology (either prior open surgical repair of an aneurysm or primary disease) and thus had associated iliofemoral disease. The absence of mortalities in this study is directly attributed, in part, to the thoroughness of the preoperative planning for vascular access and appropriate use of retroperitoneal conduits.

Prosthetic graft infection is a significant concern following repair of ABF. In open surgical repair, it is recommended that the prosthetic graft and fresh suture line be protected from the repaired bronchial fistula by either wrapping the graft with aneurysmal wall or interposing viable tissue such as pleura, intercostal muscle pedicle flap, omentum, or pericardial fat pad. ${ }^{7,11}$ Antibiotic irrigation is recommended intraoperatively, and the prosthetic graft can even be soaked in antibiotic solution prior to implantation. ${ }^{7}$ ELG repair of ABF does not allow for similar maneuvers to protect against endoprosthesis infection. In this study, there were no endoprosthesis infections. It is unclear as to why the infection rate for endovascular repair is minimal, but the ELG remains in the center of the aneurysm sac well away from the actual fistula and source of contamination. Perhaps because there is minimal tissue trauma associated with the deployment of the ELG, as opposed to open surgical repair, the excluded aneurysmal or pseudoaneurysmal cavity is much less likely to become contamination or infected. Additionally, the excluded aneurysmal wall and cavity may still be vascularized and remain viable, to some degree, after endovascular repair from fine collaterals branches that are not disrupted with deployment of the ELG. All patients received 3 intravenous doses of perioperative cephalosporin antibiotics. Subsequently, no routine postoperative antibiotic regimen was followed. In fact, 2 patients received no postoperative antibiotics and were discharged home without any oral antibiotics. In this limited cohort, there does not appear to be an association between postoperative antibiotic administration and freedom from graft infection. Additional studies are needed before definitive recommendations for antibiotic administration can be generated. In the meantime, we feel it is appropriate to discharge the patients with 1 week of outpatient oral antibiotics.

This study has several limitations. First, it is a small series and has associated limitations for making broader generalizations. However, the results are promising for an endovascular approach to $\mathrm{ABF}$ when taken in the context of the additional 36 patients reported in the world's literature. Second, this is a retrospective review and a subset of patients taken from a larger series of thoracic endografting patients from a single center. To better understand the risks and benefits of an endovascular approach, a prospective, randomized study is needed. However, it is unlikely that such a study can be conducted due to the rarity of ABF and the limited number of cases. Most likely, any conclusions that will be drawn will be based upon a meta-analysis of the literature and from large-scale multicenter national research trials for endovascular devices in general. Finally, although the follow-up for patients with ABF treated with an ELG extends up to 6.25 years in this study, the mean follow-up is $42.6 \pm 28.5$ months. Currently, all patients are alive, and continued surveillance of these patients is critical. There is potential for recurrence of the fistula, as 1 of the patients developed an ABF 6 years following placement of a thoracic ELG. It will be important to assess any late graft infections, device failures, or migrations or adverse patient outcomes. 
In conclusion, this study demonstrates that it is possible to treat patients with $\mathrm{ABF}$ using an endovascular approach and that the results compare favorably with open surgical repair. Patients tolerated the procedure well, and hemoptysis resolved in all 7 patients. There were no deaths, no episodes of paraplegia, and no endoprosthesis infections. All patients are still alive and there have been no reinterventions. Careful preoperative planning is essential for optimal results when patients are being considered for endovascular repair, especially with regards to vascular access considerations. In our cohort, duration and type of postoperative antibiotics does not appear to correlate with freedom from graft infection. Although endovascular technologies are emerging for the treatment of many types of aortic pathologies, significant questions relating to this therapeutic modality are yet to be resolved including long-term device durability, improved long-term patient outcomes, and economic concerns of increased device cost and expenses related to long-term graft surveillance with CT. Additional investigation and continued graft surveillance are critical to help determine the future role of endovascular technologies.

\section{References}

1. Orend KH, Scharrer-Pamler R, Kapfer X, Kotsis T, Gorich J, SunderPlassmann L. Endovascular treatment in diseases of the descending thoracic aorta: 6-year results of a single center. J Vasc Surg. 2003:37: 91-9.

2. Thompson CS, Ramaiah VG, Rodriguez-Lopez JA, Vranic M, Ravi R, DiMugno L, et al. Endoluminal stent-graft repair of aortobronchial fistulas. J Vasc Surg. 2002;35:387-91.

3. Kimura N, Kawahito K, Murata S, Yamagushi A, Adachi H, Ino T. Aortobronchial fistula resulting from a mycotic pseduoaneurysm after treatment of an aortoesophageal fistula due to a thoracic aortic aneurysm. Jpn J Thorac Cardiovasc Surg. 2005;53:619-23.

4. Islam S, Williams DM, Teitelbaum DH. Aortobronchial fistula from invasive Aspergillus infection of the lung: an endovascular approach to repair. J Pediatr Surg. 2005;40:e19-22.

5. Hoff SJ, Johnson SJ, Frist WH. Aortobronchial fistula after unilateral lung transplantation. Ann Thorac Surg. 1993;56:1402-3.

6. Masjedi MR, Davoodian P, Forouzesh M, Abttahi SJ. Broncho-aortic fistula secondary to pulmonary tuberculosis. Chest. 1988;94:199-200.

7. Eren E, Keles C, Toker ME, Ersahin S, Erentug V, Guler M, et al. Surgical treatment of aortobronchial and aortoesophageal fistula due to thoracic aortic aneurysm. Tex Heart Inst J. 2005;32:522-8.

8. Numan F, Arbatli H, Yagan N, Demirsoy E, Sonmez B. Endovascular treatment of an aortobronchial fistula. Cardiovasc Intervent Radiol. 2004;27:71-3

9. Munneke G, Loosemore T, Smith J, Thompson M, Morgan R, Belli AM. Pseudoaneurysm after aortic coarctation repair presenting with an aortobronchial fistula successfully treated with an aortic stent graft. Clin Radiol. 2006;61:104-8.

10. Khare RK, Settimi PD, Mba NI, Wechsler DS, Bratton SL, Williams DM. Aortobronchial fistula in a pediatric patient with massive hemoptysis: treatment by means of an aortic endograft. Ann Thorac Surg. 2005;80:731-3.

11. Piciche M, DePaulis R, Fabbri A, Chiariello L. Post-operative aortic fistulas into the airways: etiology, pathogenesis, presentation, diagnosis and management. Ann Thorac Surg. 2003;75:1998-2006.

12. Liu SF, Chen YC, Lin MC, Kao CL. Thoracic aortic aneurysm with aortobronchial fistula: a thirteen-year experience. Heart Lung. 2004; $33: 119-23$.
13. Campagna AC, Wehner JH, Kirsch CM, et al. Endovascular stenting of an aortopulmonary fistula presenting with hemoptysis. A case report. J Cardiovasc Surg (Torino). 1996;37:643-6.

14. Chuter TA, Ivancev K, Lindbland B, Brunkwall J, Aren C, Risberg B. Endovascular stent-graft exclusion of an aortobronchial fistula. $J$ Vasc Interv Radiol. 1996;7:357-9.

15. Karmy-Jones R, Lee CA, Nicholls SC, Hoffer E. Management of aortobronchial fistula with an aortic stent-graft. Chest. 1999;11:255-7.

16. Miyata T, Ohara N, Shigematsu H, et al. Endovascular stent graft repair of aortopulmonary fistulas. J Vasc Surg. 1999;29:557-60.

17. Dorweiler B, Dueber C, Neufang A, Schmiedt W, Pitton MB, Oelert $\mathrm{H}$. Endovascular treatment of acute bleeding complications in traumatic aortic rupture and aortobronchial fistula. Eur J Cardiothorac Surg. 2001;19:739-45.

18. Smayra T, Otal P, Soula P, Chabbert V, Cerene A, Joffre F, et al. Pseudoaneurysm and aortobronchial fistula after surgical bypass for aortic coarctation: management with endovascular stent-graft. $J$ Endovasc Ther. 2001;8:422-8.

19. Ciati JM, Marin ML, Flores RM, Smith CR, Martin EC, Todd GJ. Endovascular management of an aortobronchial fistula arising after resection of a primary aortic sarcoma: a case report. Vasc Surg. 2001;35:73-9.

20. Yoo JH, Lee CT, Shim YS, Chung JW, Ahn H, Kim KW. Aortobronchial fistula presenting as recurrent hemoptysis and successfully treated with an endovascular stent graft. Respiration. 2001;68:537-9.

21. Kramer S, Pamler R, Seifarth H, Brambs HJ, Sunder-Plassmann L, Gorich J. Endovascular grafting of traumatic aortic aneurysms in contaminated fields. J Endovasc Ther. 2001;8:262-7.

22. Leobon B, Roux D, Mugniot A, Rousseau H, Cerene A, Glock Y, Fournial G. Endovascular treatment of thoracic aortic fistulas. Ann Thorac Surg. 2002;74:247-9.

23. Pitton MB, Schmiedt W, Neufong A, Herber A, Duber C, Thelen M. Emergency endovascular treatment of acute aortic diseases. Rofo. 2002;174:593-9.

24. Kochi K, Okada K, Watari M, Orihashi K, Sueda T. Hybrid endovascular stent grafting for aortic arch aneurysm with aortopulmonary fistula. J Thorac Cardiovasc Surg. 2002;123:363-4.

25. Bockler D, Schumacher H, Schwarzbach M, Ockert S, Rotert H, Allenberg JR. Endoluminal stent-graft repair of aortobronchial fistulas: bridging or definitive long-term solution? J Endovasc Ther. 2004;11: 41-8.

26. Thalhammer A, Balzer J, Doss M, Jacobi V, Vogl T. Thoracic aortic stenting: indications and results. Hamostaseologie. 2004;24:157-61.

27. Kaw LL, Owens EL, Kansal N. Endovascular repair of an aortopulmonary fistula via the axillary artery. Ann Vasc Surg. 2005;19:487-91.

28. Hausegger KA, Oberwalder P, Tiesenhausen K, et al. Intentional left subclavian artery occlusion by thoracic aortic stent-grafts without transposition. J Endovasc Ther. 2001;8:472-6.

29. Wheatley GH, Gurbuz AT, Rodriguez-Lopez JA, Ramaiah VG, Olsen D, Williams J, et al. Midterm outcome in 158 consecutive Gore TAG thoracic endoprostheses: single center experience. Ann Thorac Surg. 2006;81:1570-7.

\section{Discussion}

Dr Craig Miller (Stanford, Calif). Thank you, Dr Gelfand and Dr Hawkins. Grayson, that was a very nice presentation that we've come to expect from you.

This is the third report from the Arizona Heart Institute on endovascular treatment of aortotracheal fistulas. Before you got there, there was a report in 2002 with 2 cases. In your large total experience reported this year in the Annals you had 4, and now you have taken us up to 7. You pointed out in the paper that there are 36 cases in the literature and now with 3 more additional, so 39, with an overall mortality rate of $8 \%$, which is pretty darn good considering that a lot of these patients need reoperations and have had previous grafts and whatnot. I think that is very encouraging. The question, therefore, is not can it be done. You and others and we have shown that it can be done, but how effective and how 
durable is it for what can be a devastatingly bad situation? And here I think you have provided some very good news with your follow-up: $43 \pm 29$ months going out to 6 years, no late deaths, no late infections. It's almost too good to believe, but it is good news and it is very promising and we look forward to more follow-up.

I would like to turn to something that you've already broached and that's the legal and the ethical issues at foot here. You've shown us very clearly the amount of work that Dr Diethrich and the institution had to go through to get a physician-sponsored individual investigator device exemption (IDE) from the Food and Drug Administration. We have also done that, and that allowed you to treat these patients, who would otherwise not have qualified for any of the commercial IDEs. There now are a couple high-risk IDEs that cover this but they are few in number. I want to caution the audience that if you go home and try this, if you are Gore TAG certified, it is going to be off label for an aortobronchial or for an aortoesophageal fistula or even a dissection. It is going to be off label, and you have to be very, very aware that if something doesn't go perfectly well, you don't have a leg to stand on medicolegally in court. The lawyers are out there just waiting for this, and there have been all too many examples already-an abdominal aortic stent graft or a home-brew stent graft or a commercial stent graft used for aortic dissection, for examplewhere something bad happened, and you just don't have a leg to stand on legally unless you have an institutional physician-sponsored IDE like you do. That brings me to, what are you doing now? Is your physician-sponsored IDE still current, and do you enroll them in that today just to cover yourselves? That's the first question, easy one.

Dr G.H. Wheatley (Phoenix, Ariz). Yes. We applied for, and successfully received, an extension and continuation of that protocol. Patients who are being treated with aneurysms were originally included in the protocol, but now that it is Food and Drug Administration approved for aneurysms; those patients are not enrolled in the protocol, and so the protocol now only includes off-label uses in the high-risk surgical patients.

Dr Miller. So right now, you are shut down until the Food and Drug Administration rules on your extension application?

Dr Wheatley. No, it's been approved, and we have been continuously enrolling patients without interruption.

Dr Miller. Oh, it's been renewed and extended. Okay. So you're being judicious and that's important for those of you who might see one of these desperately ill patients roll in some Friday night, because even though you are trying to save their lives, the estate of the family may not see it that way later on.

Okay, 7 cases now. Three were aneurysms, 3 were false aneurysms, and 1 traumatic case. Your slide-I'm glad you showed what happened to the sac with the serial CT scans thereafter. That was one of my questions and that information I assume will now be in the paper. You had 8 patients on that slide, not 7. Can you explain where that eighth flyer got in there? Second, could you elaborate on what happened with the patient with 1 traumatic tear, who had had a previous stent graft? Was it elsewhere? Was it your place? And why that 1 created the complication? And third, I was a little surprised to see that you did not have any giant aortic penetrating ulcers, atherosclerotic ulcers, which can cause an aortotracheal fistula. Any comments there?
Dr Wheatley. Yes. Thank you, Dr Miller, for your comments and certainly thank you for your comments regarding the manuscript and we greatly appreciate your insight.

In terms of the eighth one, that was not included in the graph. It was on the label but it wasn't there. If you count the actual lines, there were only 7. It was a benefit of PowerPoint presentations.

Interesting case, I agree. I think that we need to be very cognizant, and this is 1 of the first that I have been aware of where a patient was 3 years out from a prior ELG repair of a transection. This patient was badly injured. He had motor vehicle accident, liver laceration, and brain injury and was hemodynamically unstable, that's why he was treated with an endoluminal graft, because of his instability and very significant injury, but did well. Three years later he came back with an area distal to that ELG, which was an ulcerative lesion-no real penetrating ulcer, but there is communication right at the distal end of the graft, perhaps maybe because the graft is eroding. The patient did not have aneurysmal disease. Maybe there are some long-term consequences of the ELGs that we are not seeing, but certainly I think that this highlights that we need to be very scrupulous in following these patients, and perhaps it may be a long-term complication of ELG repair in traumatic transections.

Dr Miller. But it wasn't frankly eroded through to the outside.

Dr Wheatley. No, there was just a communication there that perhaps may have been related to the graft.

Dr Miller. And it probably wasn't atherosclerotic disease because this patient sounds like the typical trauma victim-young, healthy, tiny normal aorta and no insurance, right?

Dr Wheatley. Yes, sir.

Dr Miller. You purposely covered the left subclavian once without embolizing it or doing a transposition. You, of course, are setting yourself up for a type 2 leak and we all know that. Some of us have done it. No leak occurred in that patient. What is your current practice? Do you leave a patent left subclavian transmitting endotension to the sac?

Dr Wheatley. We do. We just reported at (ISMICS) 2 weeks ago, our complete 360-patient experience with covering the left subclavian. We cover the left subclavian approximately a third of the time and only needed a bypass in a few patients out of those patients that had covered. If we do see a retrograde type 2 leak from the subclavian, we will then embolize it using a retrograde approach from the brachial, but we manage that usually expectantly. We will usually see that if it is a type 2 endoleak, it is a minor procedure to come back and embolize the subclavian. If there is continued sac growth, it is something that we can always do later, but it is not an emergency need to treat that.

Dr Miller. That's a good answer and probably the right posture, but it is a vexing problem because of the strokes occurring acutely when the left subclavian is covered. I won't go off on a tangent to ask you how you decide when to revascularize it in advance and when not.

Finally, your topic is "Have we gone too far?" and you're pushing the envelope. Let me take you 1 step further. How many aortoesophageal fistulas have you covered with a stent graft, which we have done, but I think we are kidding ourselves and this is futile therapy. What is your experience down in Phoenix?

Dr Wheatley. I agree and that's why I did not include these in the paper. It would be a natural fit to talk about aortoesophageal 
fistulas but we have covered 3 , and 2 out of 3 have gotten infected. I really don't think that is a good approach, and that's why I did not break these patients out, but really I don't think that should be even tried.

Dr Miller. Got reinfected and died like a dog run over in the street.

Dr Wheatley. Yes, sir.

Dr Miller. Yeah, I'm afraid we echo that. Thank you for coming to the Western Thoracic.
Dr Wheatley. Thank you.

Dr Robert Cerfolio (Birmingham, Ala.). I had 2 questions but we only have time for just 1 . If you were going to cover a tracheal fistula with an aortic graft, do you now recommend that you put in a graft long enough that you go past or distal to the carina? My concern would be that the distal end of that stent is going to erode into either the left main stem or the carina unless you go past it. Is this your recommendation now?

Dr Wheatley. Yes. 